Volver a leer las prácticas. Michel Foucault, otras lecturas posibles. Páginas 269-280 en Revista de la

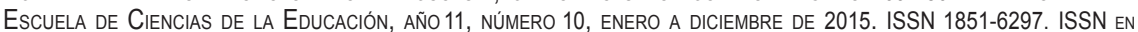
LINEA 2362-3349.

\title{
VOLVER A LEER LAS PRÁCTICAS. MICHEL FOUCAULT, OTRAS LECTURAS POSIBLES
}

\author{
Por María del Carmen Castells* \\ Universidad Nacional de Entre Ríos, Argentina. \\ mariqui_c@yahoo.com.ar
}

Recibido: 04/07/2014 Aceptado: 26/08/2014

\section{Resumen}

Con la intención de revisar nuestros marcos de lectura de las prácticas en los ámbitos de la formación docente universitaria, el presente trabajo realiza un recorrido en trazos por escritos de Michel Foucault. Indaga algunas de las aristas con que el autor aborda la noción de "prácticas" e intenta dar lugar a preguntas para leer la historicidad de las prácticas educativas y de enseñanza en nuestro presente.

En el comienzo del escrito señalamos someramente algunos obstáculos del campo de la formación docente universitaria y de determinados discursos del campo pedagógico didáctico. Desde allí se destacan, en colaboración con la lectura de una selección de textos del autor francés, aquellos puntos que nos remiten a desanclajes, a discontinuidades, a fallas entre prácticas discursivas y no discursivas, entre las prácticas de saber y de poder. Se entiende que estos giros habilitan otras lecturas de las prácticas educativas, donde cabe no ya el buscar coherencias, sino adentrase en tensiones y en disrupciones que permitirían comprender los escenarios contemporáneos de las prácticas educativas.

\section{Palabras clave:}

Prácticas - Lectura - Michel Foucault - Formación docente.

\footnotetext{
* Prof. Adjunta Ordinaria a cargo de Didáctica I - Facultad de Ciencias de la Educación (UNER). Asesora Pedagógica - Facultad de Ingeniería Química (UNL). Categoría II de Investigación. Magister en Educación y doctoranda del Doctorado en Educación de la misma Universidad. Docente de la Maestría en Educación, UNER, en sede Tierra del Fuego.
} 
Revista de la Escuela de Ciencias de la Educación, año 11, número 10, enero a diciembre de 2015. Páginas 269-280. ISSN 1851-6297. ISSN en LINEA 2362-3349. Volver a LeER LAS PRÁCticAS. MiCHEL FouCAult, OtRAS LECTURAS POSIBLES. María del Carmen Castells

\begin{abstract}
In order to check over our reading frames of the practices in the areas of university teacher education, this paper performs a dashed path through the written of Michel Foucault. It explores some of the edges in which the author discusses the notion of "practices" and intends to raise questions for reading the historicity of educational and teaching practices in our present.

In the beginning of written we briefly outlined some obstacles in the field of university teacher training and of certain discourses of didactic pedagogical field. From there, in collaboration with the reading of a selection of texts by French author, stand out those items that refer us to dislocations, discontinuities and failures both between discursive and non-discursive practices and like between the knowledge and power practices. It is understood that these turns enable other readings of educational practices, where it is no longer the search for coherence, but to penetrate in tensions and disruptions that would allow understand the contemporary scenes of the educational practices.
\end{abstract}

\title{
Keywords:
}

Practices - Reading - Michel Foucault - Teacher training.

La invitación a volver a "leer las prácticas" supone dimensionar la complejidad de esta tarea. Máxime, cuando reconocemos que los horizontes de análisis, de enunciación y de experiencia en el campo pedagógico-didáctico están atravesados por sesgos que nos conducen, en algunos casos, a interpretaciones clasificatorias apresuradas y, en otros, a enunciaciones que no siempre dan cuenta de sus razones históricas, de sus condiciones de emergencia y de sus singularidades. Desde allí, es frecuente observar que, muchas de las intervenciones provenientes del espacio mencionado, no alteran nuestros puntos de vista pues se reafirman en análisis confirmatorios de supuestos previos.

Muchas veces hallamos que ciertas propuestas de formación docente en la Universidad, referidas a prácticas de enseñanza ubicadas en ámbitos institucionales externos a ella, instan a realizar análisis desde lógicas de lo ya teorizado y esperan encontrar coherencias o "sacar a la luz incoherencias". La pretensión de buscar en las prácticas una transparencia de la conciencia de los sujetos que las efectúan nos coloca en posiciones que inevitablemente deslizan rasgos de simplificación, a la vez que de omnipotencia. Tal pretensión refleja, como en un juego de espejos, dificultades para reconocer que las propias prácticas como la de los otros reúnen también niveles de incoherencias, de conflictividad, de repetición y de justificaciones nunca plenamente conscientes.

Diferentes discursos acerca de las prácticas, que aquí no viene al caso enumerar, significaron y tuvieron efectos en los modos en que concretamos las relaciones entre las prácticas de teorización, las prácticas de educación y las de enseñanza. En estos modos advertimos improntas que, en ocasiones, hacen perder de vista distancias y diferencias así como tensiones, dispersiones/ 
Revista de la Escuela de Ciencias de la Educación, año 11, número 10, enero a diciembre de 2015. Páginas 269-280.

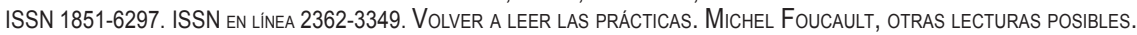
María del Carmen Castells

juegos de fuerzas que acontecen entre prácticas diversas (Bourdieu,1991). Entendemos que también allí se impide reconocer lo que se pone en juego en la producción de prácticas discursivas y en sus pretensiones de regulación, tanto de las prácticas educativas como de sus lecturas. Así mismo, perspectivas de análisis y temáticas construidas a partir de investigaciones se han transpuesto en la organización y en la concreción de trabajos prácticos para la formación universitaria de profesores. Dichos pasajes, si bien facilitaron y facilitan interacciones con experiencias educativas que no son las propias, dejan al descubierto otras dificultades. Entre ellas, la conformación de vínculos entre docentes y estudiantes concretos en las distintas instituciones desde una apertura a sus historicidades, sus conflictividades, sus heterogeneidades.

Ante eso y ante las profundas transformaciones de las que somos partícipes en el campo social amplio y por ende en el campo educativo, nos sentimos movilizados a revisar perspectivas de trabajo académico y político, a interrogar nuestras prácticas y las lecturas que de ellas realizamos y promovemos realizar, conscientes que, por su opacidad y rutina, devienen invisibles. En este sentido, es prioritario en la Universidad gestar espacios de reflexividad compartida también con autores que habiliten la emergencia, no ya de lo que confirma nuestras referencias teóricas sino de lo que las disloca y habilita pensar lo no pensado. Volver preguntarnos por las prácticas en la formación académica de los docentes conduce a interrogar los órdenes enunciativos en los que estamos inscriptos, los protocolos de lecturas que proponemos. Las prácticas, tanto en la formación académica como en la profesional, delinean transversalidades que diseminan sus sentidos y escapan a posibles identificaciones reflejas, pero también muestran en experiencias vitales, co-existencias con lo instituido que pretende imponerse en términos totalizantes.

\section{Michel Foucault: aportes para releer las prácticas. Breves comentarios.}

Sabemos por Michel Foucault que el comentario es otra forma de orden de los discursos: concreta recortes, nuevas jerarquizaciones, subsunciones, sobre la producción textual de los creadores. Bajo esa advertencia, lo que proponemos a continuación no es más que una reescritura en trazos de algunos de los textos del filósofo, a propósito del problema que nos convoca.

Los diversos desplazamientos conceptuales realizados por el autor que, de manera permanente nos obligan a cambiar de focos de atención, hacen que la lectura de sus textos se asemeje a habitar laberintos borgeanos en los que siempre y a cada paso estamos en el mismo y en otro lugar. Su lectura nos condena no sólo a reconocer la historicidad de los temas que él asume, sino a adentrarnos en el discurrir de su escritura y en sus múltiples identidades, en la de sus elecciones temáticas, en las transformaciones constantes de los ejes y sus modos de análisis, en las transmutaciones permanentes que intenta explicitar, cada tanto, en las memorias de su propio trabajo. 
Revista de la Escuela de Ciencias de la Educación, año 11, número 10, enero a diciembre de 2015. Páginas 269-280.

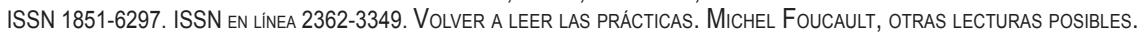
María del Carmen Castells

Si tomamos como un atajo posible la preocupación que atraviesa la vida intelectual de Michel Foucault de realizar una "ontología de nosotros mismos", en tanto crítica histórica de los modos en los que llegamos a ser lo que somos, indagar sobre la noción de prácticas -discursivas y no discursivas- nos remite a aquellos procedimientos históricos, contingentes, por los cuales nos constituimos como sujetos de saberes, de poderes y de la ética. (Foucault, 1996).

La centralidad final atribuida al concepto de práctica/s, -que no designa la actividad de un sujeto, sino la materialidad de las regularidades por las que un sujeto deviene en tal-, pone en evidencia, sin duda, las articulaciones entre saber y poder que siempre planteó en su trabajo: poderes que producen saberes, poderes y saberes que producen objetos, sujetos y políticas de verdad.

La analítica de Foucault no explora representaciones, mentalidades o condiciones superestructurales determinantes de las acciones de los hombres sin que ellos lo sepan. Tampoco implica una búsqueda para encontrar en las prácticas intenciones tácitas o explícitas que las guían. Por fuera de todo análisis hermenéutico/interpretativo sus estudios nos brindan aproximaciones a las condiciones y a los modos en que ciertas prácticas se configuran, se regularizan, se estabilizan, resisten o se diversifican y se diseminan a partir de convergencias y dispersiones de fuerzas plurales que producen sujetos y políticas de verdad.

El autor abre, en las propias fisuras de determinados conjuntos prácticos, una ruta de análisis que recorre tanto estratos como estrategias que van produciendo nuevas emergencias de prácticas. Es, desde problematizaciones constantes a las que las entiende como irrupciones que alteran los juegos de verdad y de falsedad de formaciones establecidas, donde estos análisis encuentran su lugar (Foucault, 1991, p. 232). Por debajo de poderes conclusos rastreará la conjunción entre formaciones discursivas y no discursivas y delimitará transversalmente los espacios y las condiciones donde se ejercen y aparecen.

\section{Prácticas discursivas}

Las prácticas discursivas no son pura y simplemente modos de fabricación de discursos. Ellas toman cuerpo en el conjunto de técnicas, de las instituciones, de los esquemas de comportamiento, de los tipos de transmisión y de difusión $\mathrm{y}$ de las formas pedagógicas que a la vez imponen y las mantienen (Dichos y Escritos, 241. En Castro, E., 2004, p. 94).

Para Foucault el lenguaje es acción porque no se limita a la frase o a la palabra dicha. Los enunciados que componen el discurso foucaultiano no son solo una reunión de proposiciones cuyo contenido, contexto de producción o validez lógica habrá que indagar. La analítica que este autor emprende de las prácticas discursivas, pone en evidencia la propia fuerza productiva que el lenguaje ostenta, porque deshace de manera definitiva el nexo entre éste y 
Revista de la Escuela de Ciencias de la Educación, año 11, número 10, enero a diciembre de 2015. Páginas 269-280. ISSN 1851-6297. ISSN en LINEA 2362-3349. Volver a Leer LAS PRÁCticAs. MiChel FouCAult, OtRAS LECTURAS POSIBLES. María del Carmen Castells

la cosa. Este desacople que plantea una ruptura con la correspondencia o la representación y con la referencia a un significado y al sujeto de conciencia, nos coloca frente a la densidad del lenguaje y a su función efectiva en la producción de marcas, de rastros en las prácticas sociales y en las políticas de verdad. La verdad deja de buscarse en una transparencia para encontrarse en los efectos que se ejerce como práctica en las prácticas y, ya no será la que propone el sujeto consciente, sino que será la de un juego que delinea formas, "cuadros", donde pueden leerse emergencias enunciativas y visibilidades, trazos transversales que esbozan posiciones de sujetos, delimitaciones de objetos, problematizaciones.

(...) La propuesta de la arqueología foucaultiana es pervertir la inquietud epistemológica moderna e instalar una nueva inquietud. En lugar de preocuparse por hallar criterios que permitan distinguir los discursos legítimos, volver la mirada hacia esos monumentos discursivos en torno a los cuales se despliegan las problematizaciones que organizan las prácticas colectivas. En vez de demarcar espacios en los que sería posible encuadrar -sin contradicciones- los códigos de la cultura, recorrer las líneas enunciativas que alimentan las interpretaciones de la experiencia. En lugar de indagar las condiciones de posibilidad de un discurso válido, indagar las estrategias que deciden la emergencia de las 'cosas dichas', su conservación, su transformación" (Britos, 2003, p.3).

La tarea arqueológica se reconoce enredada en la propia historia del lenguaje, habitada por ella. Sin embargo al mismo tiempo intenta, en 'una actitud deliberada' que despega al lenguaje de su "función al servicio del significante", despojarse de toda la clasificatoria que la ordena desde categorías de unidad, totalidad y continuidad o de otra sucesión de señalamientos que refieren al orden interno de los discursos. Estas operaciones de la arqueología nos colocan frente "lo efectivamente dicho". No se detienen en análisis internos del decir y de lo dicho, sino que ponen al descubierto en las prácticas discursivas su ejercicio de control en un fondo desordenado del lenguaje y propone una lectura que, desde la categoría de discontinuidad, conecta los discursos con pasados múltiples, con encadenamientos no explorados, con relaciones entre saberes y fuerzas no transitadas. Así, en lugar de tratar acerca de proposiciones y frases, Foucault recorre enunciados y formaciones discursivas que pueden leerse como emergencias acontecimentales móviles: se revelan en sus regímenes de producción de objetos, de sujetos, de verdades; regularidades, estabilizaciones, estratos, a la vez que en anudamientos de sentidos plurales que nos remiten a lo que es posible decir y ver en una época determinada.

Es entonces, desde un análisis externo donde la productividad discursiva se evidencia en sus movimientos estratégicos que delinean relaciones entre 
Revista de la Escuela de Ciencias de la Educación, año 11, número 10, enero a diciembre de 2015. Páginas 269-280. ISSN 1851-6297. ISSN en LINEA 2362-3349. Volver a LeER LAS PRÁCticAS. MiCHEL FouCAult, OtRAS LECTURAS POSIBLES. María del Carmen Castells

"superficies de emergencias", "instancias de delimitación", "rejillas de especificación". Relaciones que no se suturan universalmente en todas las problemáticas que el filósofo aborda: en cada caso asumen una especificidad histórica y por ello exigen una analítica particular que capta sus desplazamientos, sus quiebres, sus rearticulaciones y dispersiones que rompen toda posibilidad de pensar los discursos como formaciones estáticas.

\section{Poder}

No hay relaciones de poder que triunfen por completo y cuya dominación sea imposible eludir" (Foucault, 2012, p. 77). (...) Si las relaciones de poder produjeron formas de investigación, análisis de los modelos del saber, fue precisamente porque el poder no era omnisciente sino ciego, porque estaba en un callejón sin salida. Si se ha constatado el desarrollo de tantas relaciones de poder, de tantos sistemas de control, de tantas formas de vigilancia, fue precisamente porque el poder sigue siendo impotente" (Foucault, 2012, p.117).

Ya sabemos que el problema del poder es insoslayable en este análisis de las prácticas y, para transitar este camino, Foucault fija una condición que será conceptual y desde luego metodológica: deshacer las concepciones mecánicas de las relaciones de poder por las cuales entendemos que el mismo es siempre vertical, represor, negativo, prohibitivo; tampoco se encuentra en algún lugar de concentración total y definitiva. Su opción conceptual será nominalista y, tal como expresa en La voluntad de saber, el poder es "...el nombre que se presta a una situación estratégica compleja en una sociedad dada." (Foucault, 2008, p. 89). Es un campo de relaciones de fuerzas, sin ubicación determinada, inestable, en el que más que posiciones binarias o especulares de dominación y de resistencia se despliega en puntos, donde poder y resistencia se ejercen, se necesitan, se intercambian y transmutan o se complementan para existir. Es constitutivo de toda práctica y, a la vez que la concentra, la descentra o la dispersa en diferentes sitios del espacio social, en diagramas que como espacios de juegos de fuerzas plurales, produce efectos múltiples y diversos.

En un sentido, que no se ampara entonces en la concepción jurídica del poder, es oportuno remitirnos a los conceptos de estrategias y de tácticas que el autor despliega como tecnologías de producción de prácticas. Así, a la vez que nos encontramos frente a lo que él llama "conjuntos prácticos" en tanto dominios homogéneos, nos enfrentamos a los modos con los que los hombres "... organizan modos de obrar (lo que se podría llamar su aspecto tecnológico); y la libertad con la actúan dentro de esos sistemas prácticos, reaccionando ante lo que hacen los demás, modificando hasta cierto punto las reglas del juego (es lo que podría llamar el aspecto estratégico de esas prácticas)..." (Foucault, 1996, p. 108). 
Revista de la Escuela de Ciencias de la Educación, año 11, número 10, enero a diciembre de 2015. Páginas 269-280.

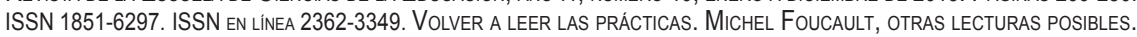
Maria del Carmen Castells

De esta manera, las preguntas por determinadas prácticas abandonan los vínculos de equivalencias con una concentración del poder, con la de la ley, con la de la obediencia, para detenerse en preguntas que reenvían a relaciones "móviles". El poder, como relación de fuerzas, aparece disperso, se ejerce sobre todo a través de procedimientos difusos que se multiplican aquí y allá, en los aparatos del Estado al igual que en los juegos que juegan sujetos comunes. Sin lugares inequívocos y sin considerar que de un lado están los dominantes y del otro los dominados, se despliegan transversalmente estrategias que ejercen efectos performativos: incita, arrastra, resiste, sostiene, rechaza, reparte, une, distribuye, incluye, excluye, estrecha, se alinea y coopta fuerzas dispersas, a la vez que las disemina, las reproduce o las altera. Como para Foucault no hay poder absoluto, tampoco habrá éxito absoluto de una práctica de dominación.

En esta dirección, en vez de enfocar posiciones fijas -quién o quiénes detentan el poder y quién o quiénes están despojados de él-, explora las prácticas como espacio de relaciones variadas, de desplazamientos permanentes, de virajes de sentido, en las intervenciones en y las producciones de nuevos objetos, campos de saber, nuevos sujetos y verdades, inscriptos en "estrategias de conjunto". Pues si bien, ninguna práctica es refleja de otras que la subsumen, tampoco existe aislada de aquellas con las que se complementa o resiste, con las que estrecha líneas de sentidos y desde las que deviene necesaria para sostener otras prácticas en afán de hegemonizarse.

También su analítica procede de forma inversa de aquellos tratamientos que conciben las prácticas como derivaciones de las teorías o, de los discursos que las esbozan. "Discursos" y "prácticas" dejarán de ser vistos como bloques compactos, coherentes, especulares, para ser analizados en sus inestabilidades, en sus dispersiones, en sus incongruencias o en sus alianzas puntuales, $-a$ veces efímeras, a veces estables-, con juegos hegemónicos de poder.

\section{Tácticas y estrategias}

(...) Si la sexualidad se constituyó como campo a conocer, tal cosa sucedió a partir de relaciones de poder que la instituyeron como objeto posible; y si el poder pudo considerarla un blanco, eso ocurrió porque técnicas de saber y procedimientos discursivos fueron capaces de sitiarla e inmovilizarla. Entre técnicas de saber y estrategias de poder no existe exterioridad alguna (Foucault, 2008, pp.94-95).

Puestas en esta perspectiva, a las nociones de tácticas y de estrategias -utilizadas a lo largo de los escritos de Foucault para remitirse a las prácticas discursivas como no discursivas- habrá que entenderlas como movimientos heterogéneos que efectivizan condiciones para la existencia de determinadas prácticas. Así, los tratamientos foucaultianos de la locura, de la sexualidad o 
Revista de la Escuela de Ciencias de la Educación, año 11, número 10, enero a diciembre de 2015. Páginas 269-280. ISSN 1851-6297. ISSN en LINEA 2362-3349. Volver a LeER LAS PRÁCticAS. MiCHEL FouCAult, OtRAS LECTURAS POSIBLES. María del Carmen Castells

de la prisión entre otros, pusieron en clave histórica las transformaciones del ejercicio del poder a partir de los cuales ya no pueden leerse como designaciones voluntarias de actos de razón o recortes efectuados desde poderes centrales, sino como juegos de fuerzas diseminadas que, en una sociedad y en una época dada, se conjugan contingentemente.

Las nociones de tácticas y de estrategias, -que Foucault utiliza para abordar la constitución de las sociedades modernas diferentes a las medievales-, se articulan, en ocasiones con el disciplinamiento próximo a los significados que detentan las prácticas militares, otras veces con el control, pero siempre las estrategias encerrarán una economía de las prácticas porque estiman el cálculo de los efectos y de resultados de la aplicación de fuerzas o de diversas tecnologías -de producción de saberes, de sujetos, de objetos-.

También encontramos la noción de estrategia como constituyente del constructo dispositivo. Éste, que integra elementos discursivos como no discursivos, supone una formación

(...) que, en un momento histórico dado, tuvo como función mayor la de responder a una urgencia. El dispositivo tiene una posición estratégica dominante. Esta pudo ser, por ejemplo, la reabsorción de una masa de población flotante que a una sociedad con una economía esencialmente mercantilista le resultaba embarazosa: hubo ahí un imperativo estratégico, jugando como matriz de un dispositivo, que se fue convirtiendo poco a poco en el mecanismo de control-sujeción de la locura, la enfermedad mental, de la neurosis (Foucault, 1991, p.129).

Aunque en su Arqueología del saber Foucault se detiene en las estratificaciones y en las regulaciones que organizan las formaciones discursivas, advertimos que utiliza el término estrategia para referir a los grandes temas que atraviesan, enlazan y se desparraman temporal y espacialmente en espacios de saber. Estos temas, por caso el evolucionismo o la fisiocracia (Foucault, 2013 , p. 249), proponen sincrónica y diacrónicamente juegos diferentes para articular conceptos, métodos, modos de enunciación en cada período histórico. Esta lectura de las prácticas hace que sea imposible trazar una línea recta de continuidad en un mismo campo de saber y sí se abra la factibilidad de percibir en él discontinuidades, desplazamientos y, con ellos, nuevos modos de constitución de otros objetos. A un mismo tema, lo encontramos aquí y allá, pero desplazado en sus significados, en sus efectos y en el juego de relaciones que propone. Recordemos que el trabajo arqueológico de los saberes no se realiza identificando objetos teóricos para definir una disciplina, sino desplegando un espacio heterogéneo donde leyes de formación de objetos, de conceptos, de teorías, concurren junto a institucionalizaciones y materializaciones, a la construcción del saber. 
Revista de la Escuela de Ciencias de la Educación, año 11, número 10, enero a diciembre de 2015. Páginas 269-280. ISSN 1851-6297. ISSN en LINEA 2362-3349. Volver a LeER LAS PRÁCtICAS. MiCHEL FouCAULt, OTRAS LECTURAS POSIBLES. María del Carmen Castells

\section{Entre enunciados y visibilidades. Entre el saber y el poder.}

“...Es cierto que, según Foucault todo es práctica; pero la práctica de poder sigue siendo irreductible a toda práctica de saber..." (Deleuze, 2005, p.103).

En los parágrafos precedentes expusimos algunas líneas que nos aproximan a los conceptos de prácticas en Michel Foucault. Creemos necesario aquí avanzar en el juego de diferencias y de relaciones al que nos invita el filósofo en sus analíticas y que pueden convertirse en señalamientos valiosos para nuestras lecturas de las "prácticas".

Volvamos a las prácticas discursivas de la que ya algo habláramos. Estas prácticas en Foucault nos remiten, en articulación con prácticas no discursivas, al saber, noción que excede su identificación con los dominios de la ciencia para extenderse hacia "...la experiencia perceptiva, incluso los valores de lo imaginario, incluso las ideas de la época o los elementos de opinión común..." (Deleuze, 2005, p.79). Como práctica, el saber supone un agenciamiento entre enunciados y visibilidades, entre lo que es posible decir y lo que es posible ver en una época determinada, donde es dable reconocer la primacía del enunciado. Recordemos la variabilidad histórica del saber en tanto que organizado en formaciones/estratos en los que trasmutan los regímenes de enunciación y los modos de visibilización. Sin embargo la historia de los enunciados no es idéntica a la de las visibilidades; si bien entre ambas se dan capturas mutuas, éstas no son siempre coincidentes ni en el tiempo ni en el espacio porque entre ellas existen diferencias de naturaleza. El haz de luz que despliega lo visible -en la escuela, en la prisión, en el hospital- nunca será totalmente capturado por los enunciados, opone resistencia porque responde a una legalidad propia.

La primacía del enunciado solo se comprende en ese juego de disputas que abre en el seno del saber una distancia marcada por la no identidad entre lo que se dice y se ve, por "la irreductibilidad histórica de lo visible". Lo que Foucault propone como relación entre el enunciado y lo visibile, es "...una batalla en la que cada uno lanza una flecha al blanco del otro', pero en cuyos entrecruzamientos se alcanza una primacía del enunciado sobre la visibilidad" (Delueze, 2013 , p. 32), sin que esta primacía implique una cooptación inmediata, lineal, invariante. Con la ausencia de isomorfismo entre lo enunciable y lo visible, nos encontramos en presencia de una falla, una cesura, un residuo nunca pasible de ser totalmente dicho, descripto, porque en definitiva la sutura entre lo que se habla y lo que se ve es, como nos dice Deleuze, un sueño.

De esta manera, podemos entender que en las formaciones históricas, las prácticas agencien ciertas estabilizaciones -históricas- entre el ver y el decir, las mismas que, según Foucault, podrán analizarse desde aquellas "reglas de pasaje" que organizan "conjuntos prácticos", no exentos de juegos estratégicos en cuyo seno se crean nuevas reglas (Foucault, 1996) y donde se pone en evidencia que estabilización y homogeneidad para Foucault no son sinónimos. Si bien las prácticas discursivas regulan y estabilizan, es imposible advertir 
Revista de la Escuela de Ciencias de la Educación, año 11, número 10, enero a diciembre de 2015. Páginas 269-280. ISSN 1851-6297. ISSN en LINEA 2362-3349. Volver a LeER LAS PRÁCticAS. MiCHEL FouCAult, OtRAS LECTURAS POSIBLES. María del Carmen Castells

la homogeneidad de los enunciados y la de sus vínculos con lo visible; ellos serán siempre heterogéneos, dispersos y su estabilidad no estará dada por sus identificaciones recíprocas, sino por las reglas que harán simultáneos o alternos los pasajes que llegan a constituir una formación.

Otra diferenciación fundamental es la que se inscribe en la relación saber y poder, diferenciación que coloca nuevamente a las prácticas discursivas en el límite en sus intentos de control, de dominio, de estabilización, de regularidad, y por lo mismo las abre a la potencia del pensar.

Podríamos decir que entre saber y poder rigen las mismas irreductibilidades que entre enunciados y visibilidades. Nuevamente estamos en presencia de naturalezas diversas. Así, el poder a diferencia del saber, no es, no tiene sustancia, y por lo mismo no es localizable. Se presenta difuso sin un lugar -un no lugar-, reapareciendo aquí y allá en un encadenamiento móvil en el diagrama que escapa a las formas estables del enunciado y de las visibilidades (Deleuze, 2005, p.102). Si lo que caracteriza a las prácticas discursivas es su regularidad y estratificación, "lo otro" es su límite y catalizador; como ya dijimos, se caracteriza por no tener forma, por irrumpir como fuerza ciega y muda. En ese sentido Deleuze nos aclarará que esto "otro", el poder, no estabiliza, regula o estratifica, sino despliega estrategias que se distribuyen en puntos móviles, no ya en un "cuadro" dibujado por estratos de saber, sino en un "diagrama" configurado por una relación/dispersión de fuerzas.

Sin embargo, a pesar de estas diferencias y como acontece con los enunciados y las visibilidades, las prácticas discursivas y no discursivas se implican en "capturas recíprocas" y en "mutuas inmanencias", aunque no en correspondencias inmediatas, ni en reciprocidades transparentes. La evanescencia del poder encuentra un cauce estabilizador en las prácticas de saber, en operaciones de institucionalización que lo actualizan. Al mismo tiempo, los movimientos de integración entre lo enunciable y lo visible de las prácticas de saber solo se efectivizan cuando éstas se conectan al tercer elemento que las redistribuye. La primacía del poder sobre el saber se da

(...) porque las dos formas heterogéneas del saber se constituyen por integración y entran en una relación indirecta, por encima de su intersticio, de su 'no relación', en condiciones que solo corresponden a las fuerzas... si tratamos de determinar un corpus de frases y de textos para extraer de ellos enunciados, vemos que solo podemos realizarlo asignando los núcleos del poder (y de resistencia) de los que ese corpus depende...(Deleuze, 2005, pp.111-112).

El tercer término, entonces, y tal como fue definido -como fuerza pura, ciega y muda, como plurales relaciones de fuerzas-, será condición de posibilidad de múltiples emergencias de formaciones históricas y como tal, implica un afuera no externo a ellas mismas (pues las implican). De continuo va confi- 
Revista de la Escuela de Ciencias de la Educación, año 11, número 10, enero a diciembre de 2015. Páginas 269-280. ISSN 1851-6297. ISSN en LINEA 2362-3349. Volver a LEeR LAS PRÁCtICAS. MiCHEL FouCAULt, OTRAS LECTURAS POSIBLES. María del Carmen Castells

gurando nuevas condiciones para nuevas emergencias porque no se identifica con ninguna de ellas. Así el diagrama se configura en infinitos puntos/juegos de fuerzas que, al igual que en las prácticas de saber gestan vacíos, intersticios, que convocan el pensar.

\section{Volver a leer las prácticas}

Prácticas de hablar, prácticas de ver, ejercicio de poder, múltiples desdoblamientos que nos proponen otros órdenes y niveles que rompen nuestras prácticas de leer las prácticas, invitándonos a repensar y repensarlas. Esta lectura realizada en trazos de algunos de los planteos de Michel Foucault, nos propone disloques y aberturas.

El reconocimiento crudo de la fuerza efectiva del lenguaje, instalada en las prácticas de enunciación, nos aproxima a hacer visible los protocolos de lectura de las prácticas que ofrecemos muchas veces en la formación docente, protocolos/dispositivos que desde luego conforman tanto la propia experiencia (Larrosa, 1995) como la lectura de la experiencia de los otros. Desde aquí advertimos que dichos protocolos no implican un medio inocuo y transparente, por lo demás ingenuo, sino activo en la producción de sujetos y de subjetividades, de objetos y de políticas de verdad. Los dispositivos de lectura son ellos mismos generadores de efectos sobre las prácticas: juegos que intentan capturar lo visible y desde ellos no siempre se advierte un resto, lo que queda afuera en la tangente de lo instituido, lo que irrumpe y desordena lo ya estabilizado.

Las prácticas de subjetivación contemporáneas, los vínculos interpersonales que se plantean, las relaciones de y con los conocimientos, los nuevas relaciones entre saberes y mundo del trabajo en las configuraciones descentradas de espacio y de tiempo, trastocan las prácticas educativas y reclaman movimientos de comprensión y de enunciación que exceden los transitados. EI reconocimiento que realiza Foucault de una cierta autonomía de las prácticas, no codificadas desde ningún discurso, a la vez que el señalamiento sobre el papel coactivo de los enunciados que pretenden hacerlas inteligibles (Chartier, 2001) debería remitirnos a las propias condiciones de emergencia de nuestras prácticas de lectura. Al mismo tiempo, la no congruencia y la no identidad expresadas por Foucault reiteradamente en muchos de sus escritos, respecto tanto a los enunciados y a las visibilidades, como a los saberes y al poder, desvanecen los intentos de captar de manera lineal y homogénea las prácticas.

Entendemos que los giros propuestos por Michel Foucault ponen en el seno de las prácticas, regularidades pero también discontinuidades, habilitan una lectura de su historicidad -la de las prácticas y la de su lectura-. Nos advierten acerca de lo que irrumpe en el afuera de lo ya enunciado, llegando al centro mismo de sus estabilizaciones e institucionalidades, inventando nuevas reglas (aún no contenidas en prácticas discursivas), conformando otras relaciones 
Revista de la Escuela de Ciencias de la Educación, año 11, número 10, enero a diciembre de 2015. Páginas 269-280. ISSN 1851-6297. ISSN en LINEA 2362-3349. Volver a Leer LAS PRÁCticAS. MiChel FouCAult, OtRAS LECTURAS POSIBLES. María del Carmen Castells

de poder y, con ellas, nuevas apariciones de órdenes no explorados por los saberes institucionalizados.

Entonces, será posible plantear interrogantes que atañen a las prácticas educativas contemporáneas, algunos de los cuales apuntamos convencidos de que se trazan en el fondo de una apertura/murmullo infinitos:

¿Cómo y dónde se diseminan o se congregan las prácticas de educativas y de enseñanza frente a las formas -estado vigentes?. ¿Qué, dónde y quiénes las sostienen, cuando la forma institución- escuela desdibuja sus códigos tradicionales?. ¿Qué nuevas regularidades están ordenando las prácticas educativas y de enseñanza?. ¿Qué desplazamientos se están gestando en los dominios el saber acerca de la educación?

A sus respuestas, lejos de analizarlas como desviaciones a prescripciones curriculares o a regularidades legitimadas para el campo profesional docente, las comenzamos a leer como las verdaderas cuñas que ya son parte de nuestras propias prácticas.

\section{Referencias Bibliográficas}

- $\quad$ Bourdieu, P. (1991). El sentido práctico. Madrid: Taurus.

- Britos, M.del P. (2003). Del orden del discurso a una pragmática de lo múltiple. Revista Tópicos de la Asociación de Filosofía de Santa Fe. № 11. Santa Fe, Argentina. Doi:versión On-line ISSN 1666-485X.Tópicos n.11 Santa Fe 2003

- $\quad$ Castro, E. (2004). El Vocabulario de Michel Foucault. Bernal: Prometeo. UNQ.

- Chartier, R (1996). Escribir las prácticas. Foucault, de Certau y Marin. Buenos Aires: Manantial.

- Deleuze, G. (2005). Foucault. Buenos Aires: Paidós.

- Deleuze, G. (2013). El saber. Curso sobre Foucault. T.1. Buenos Aires: Cactus.

- Foucault, M. (2004). El orden del discurso. Buenos Aires: Tusquets. Fábula.

- Foucault, M. (2012). El poder, una bestia magnífica. Buenos Aires: Siglo XXI.

- Foucault, M (2008). Historia de la sexualidad. 1. La voluntad de saber. Buenos Aires: Ed. Siglo XXI.

- Foucault, M. (1997). La Arqueología del saber. Ciudad de México: SXXI.

- Foucault, M. (2013). ¿Qué es usted, profesor Foucault?. Buenos Aires: SXXI.

- Foucault, M. (1996). ¿Qué es la llustración?. Córdoba: Alción Editora.

- Foucault, M. (1991). Saber y verdad. Madrid: La Piqueta.

- Larrosa, J. (1995). Escuela, poder y subjetivación. Madrid: La Piqueta. 